\title{
New EUV Selected Flare Stars
}

\author{
Bill Ball, Gordon Bromage \\ Centre for Astrophysics, University of Central Lancashire, Preston PR1 2HE, UK
}

\section{Introduction}

The ROSAT Wide Field Camera all-sky EUV survey of 1990-91 identified over 200 active late-type stars (Pounds et al. 1993). Most of these were not known to be active prior to the survey and follow-up optical spectroscopy has been successful in selecting the most active dMe stars. These stars are characterized by their Balmer line emission which is direct evidence for an active chromosphere. We present photometric monitoring data for 4 new dMe stars, including the discovery of flaring activity on all 4 stars.

\section{Observations}

The observations were made at the South African Astronomical Observatory at Sutherland near Capetown in October 1994, using the $0.5 \mathrm{~m}$ and $1.9 \mathrm{~m}$ telescopes for photometry and spectrometry, respectively. The $0.5 \mathrm{~m}$ telescope is equipped with an automated, pulse-counting photometer. Flare monitoring of all the stars was done in the U-band with either 5 or $10 \mathrm{~s}$ integration times. All the stars monitored were between magnitude $V=11$ and 13 . A total of 15 flares were detected, including at least one on each star (Table 1). Comparison stars were measured in standard $U B V R I$ photometric bands for each star.

There are some unique aspects of this observing run. This is the first time that candidate stars for flare monitoring have been chosen systematically from their EUV/bolometric flux ratio. Several flares, including the largest, were seen on previously unmonitored $\mathrm{dMe}$ stars and the $4^{\mathrm{m}} 0$ flare was one of the largest optical flares to be observed since the giant $4^{\mathrm{m}} 5$ flare in 1985 on AD Leo (Hawley \& Pettersen 1991).

On the last but one night of simultaneous observing a large $4^{\mathrm{m}}$ flare was observed on the EUV selected star RE J0241-53N, with a time resolution of 10 sec (Fig. 1). This star is the northerly, fainter component of a binary dMe pair with a separation of $25^{\prime \prime}$. The flare started at 00:20 UT on $23 \mathrm{rd}$., rose $\approx 4 . \mathrm{m} 0$ in about $15 \mathrm{~min}$. and had not returned to quiescence by the end of the observations 
Table 1. Summary of flares observed in October 1994

\begin{tabular}{|c|c|c|c|}
\hline Star & $\begin{array}{c}\text { Monitoring time } \\
\text { (hours) }\end{array}$ & $\begin{array}{c}\text { Largest Flare } \\
(\Delta U)\end{array}$ & $\begin{array}{c}\text { No. flares } \\
\text { seen }\end{array}$ \\
\hline RE J0241-53N & 36 & 4.0 & 5 \\
RE J0604-34 & 9 & 2.5 & 5 \\
RE J0625-60 & 10 & 1.5 & 3 \\
RE J2055-17B & 21 & 0.3 & 1 \\
\hline
\end{tabular}
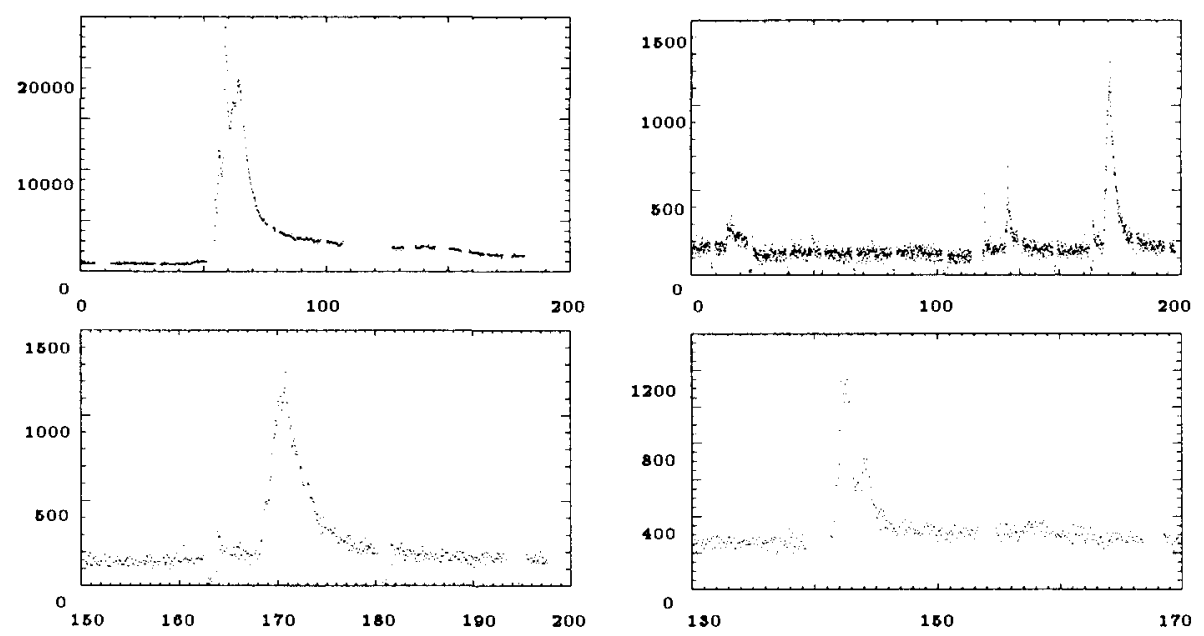

Fig. 1. Light curves of counts vs. time in min from start of monitoring on RE J0241-53N (top left), RE J0604-34 and RE J0625-60 (bottom right).

at 03:00. The breaks in the curves are due to sky readings. It can be seen that the number of counts started to increase just prior to the main flare onset. This star also flared on October 24 and 25 , rising $0^{\mathrm{m}} 5$ and $1.2 \mathrm{~m}$, respectively, and twice on October 27, rising 0.7 each time.

The star RE J0604-34 produced a sequence of events on October 31 (Figs. 1b, c). Two small short-lived flares of $\approx 0.8$ occurred at $22: 15$ and $22: 45$, followed at 23:58 by a $20 \sec 11^{\mathrm{m}} 4$ and one of $1^{\mathrm{m}} 7$ at $24: 07$. The largest flare on RE J0604$34\left(2^{\mathrm{m}} 5\right)$ occurred at $24: 48$ and again there was some preflare activity. The integration time was $5 \mathrm{~s}$ for this star.

The star RE J0625-60 was seen to flare at 22:19, 23:44 and 24:44 on October 30 (Fig. 1d), also with 5 s integration time. The largest was a $\approx 1^{\mathrm{m}} 5$ event lasting $\approx 30 \mathrm{~min}$.

Finally, a small 0.3 flare was seen on RE2055-17B at 18:32 on October 31 using 5 secs integration time.

\section{References}

Hawley S.L., Pettersen B.R., 1991, ApJ 378, 725

Pounds K.A., et al., 1993, MNRAS 260, 77 